\title{
The role of substance $P$ in acupuncture signal transduction and effects
}

\author{
Yu Fana , Do-Hee Kima ${ }^{a}$, Young Seob Gwak ${ }^{a}$, Danbi Ahn ${ }^{a}$, Yeonhee Ryu ${ }^{b}$, Suchan Chang ${ }^{a}$, \\ Bong Hyo Lee ${ }^{a}$, Kyle B. Bills ${ }^{c, d}$, Scott C. Steffensen ${ }^{c}$, Chae Ha Yang ${ }^{a}$, Hee Young Kim ${ }^{a}{ }^{*}$ \\ aDepartment of Physiology, College of Korean Medicine, Daegu Haany University, Daegu 42158, \\ South Korea \\ bKorean Medicine Fundamental Research Division, Korea Institute of Oriental Medicine, Daejeon \\ 34054, South Korea \\ 'Department of Psychology and Neuroscience, Brigham Young University, Provo, UT 84602, \\ United States \\ dDepartment of Biomedical Sciences, Noorda College of Osteopathic Medicine (P), Provo, UT \\ 84604, United States
}

\section{Abstract}

Background: Acupuncture has been used to treat a wide variety of diseases, disorders, and conditions for more than 2500 years. While the anatomical structures of acupuncture points (or acupoints) are largely unknown, our previous studies have suggested that many acupoints can be identified as cutaneous neurogenic inflammatory spots (neurogenic spots or Neuro-Sps), arising from the release of neuropeptides from activated small diameter sensory afferents at topographically distinct body surfaces due to the convergence of visceral and somatic afferents. In turn, the neuropeptides released during neurogenic inflammation may play important roles in the effects of acupuncture as well as the formation of active acupoints. Thus, the present study has focused on the role of substance P (SP) in acupuncture signal transduction and effects.

Methods: Neuro-Sps were detected by using in vivo fluorescence imaging after intravenous injection of Evans blue dye (EBD) and compared with traditional acupoints. Stimulatory effects of the Neuro-Sps were examined in a rat model of immobilization-induced hypertension (IMH). The roles of increased SP in Neuro-Sps were also investigated by using immunohistochemistry, in vivo single-fiber peripheral nerve recordings, and in vivo midbrain extracellular recordings.

*Corresponding author. hykim@dhu.ac.kr (H.Y. Kim).

Author contributions

HYK conceived and designed research. YF, DHK, YSG, DA, YR, SC, BHL and CHY performed the research. YF, KBB, SCS and HYK analyzed the data and drafted the manuscript. HYK was responsible for the overall direction of the project and for the editing of the manuscript. All authors read and approved the final manuscript.

Declaration of Competing Interest

The remaining authors declare that the research was conducted in the absence of any commercial or financial relationships that could be construed as potential conflicts of interest.

Appendix A. Supplementary data

Supplementary data to this article can be found online at https://doi.org/10.1016/j.bbi.2020.08.016. 
Results: Neurogenic inflammation quickly appeared at acupoints on the wrist and was fully developed within $15 \mathrm{~min}$ in IMH model. The Neuro-Sps showed an increased release of SP from afferent nerve terminals. Mechanical stimulation of these Neuro-Sps increased cell excitability in the midbrain (rostral ventrolateral medulla) and alleviated the development of hypertension, which was blocked by the local injection of the SP receptor antagonist CP-99994 into Neuro-Sps prior to acupuncture and mimicked by the local injection of capsaicin. Single fiber recordings of peripheral nerves showed that increased SP into the Neuro-Sps elevated the sensitivity of A- and C-fibers in response to acupuncture stimulation. In addition, the discharge rates of spinal wide dynamic response (WDR) neurons significantly increased following SP or acupuncture treatment in NeuroSps in normal rats, but decreased following the injection of CP-99994 into Neuro-Sps in IMH rats.

Conclusions: Our findings suggest that SP released during neurogenic inflammation enhances the responses of sensory afferents to the needling of acupoints and triggers acupuncture signaling to generate acupuncture effects.

\section{Keywords}

Acupuncture; Neurogenic inflammation; Substance P; Sensory afferents; Wide dynamic response neurons; Rostral ventrolateral medulla

\section{Introduction}

Acupuncture originated in China more than 2500 years ago and is one of the oldest medical procedures in the world. Acupuncture involves penetrating the skin with thin solid metal needles at specific locations called acupuncture points or acupoints (Stux and Pomeranz, 1987). Traditional Chinese medicine describes how each acupoint communicates with a specific visceral organ; an acupoint reflects the status of a visceral organ, and internal disorders can be treated by stimulating the acupoints (Rong et al., 2011; Stux and Pomeranz, 1987). Currently, acupuncture therapy is widely accepted and practiced for the treatment of a variety of diseases, including visceral disorders and pain conditions.

Substance $\mathrm{P}(\mathrm{SP})$ is encoded by the tachykinin precursor 1 gene and belongs to the tachykinin family of peptides, and it is broadly distributed in the central and peripheral nervous systems (Mantyh, 2002; Martinez and Philipp, 2016). Substance P has a high affinity for the neurokinin 1 receptor (NK1R), to which it binds preferentially (Corrigan et al., 2016; Douglas and Leeman, 2011). Due in part to its broad anatomical distribution, the $\mathrm{SP} / \mathrm{NK} 1 \mathrm{R}$ system is involved in a variety of complex physiological responses, including neuroinflammation, microvascular permeability, and pain (Martinez and Philipp, 2016). Additionally, some studies have shown that the modulation of SP binding to NK1R may be useful in the treatment of diseases, such as inflammatory conditions associated with the gastrointestinal tract, emesis, depression, cancer, and social anxiety disorder (Garcia-Recio and Gascón, 2015; Huang and Korlipara, 2010).

Visceral diseases frequently produce a referred pain at distant somatic sites due to the viscerosomatic convergence of sensory afferent pathways. In multiple sites of skin overlying the referred pain, local tissue responses, known as neurogenic inflammation (neurogenic spots or Neuro-Sps), are observed (Wesselmann and Lai, 1997). Our previous study showed 
that Neuro-Sps share the characteristics of acupoints (Kim et al., 2017). Our previous studies have suggested that neuropeptides, including SP, contribute to the physiological features of acupoints in disease. Under pathological conditions, such as hypertension or colitis, the skin over acupoints exhibits neurogenic inflammation (Kim et al., 2017, 2006) characterized by vasodilation and vascular leakage (plasma extravasation) arising from the release of the neuropeptides SP and calcitonin gene-related peptide (CGRP) from activated small diameter sensory afferents (Schmelz and Petersen, 2001). We also demonstrated that high electrical conductance at acupoints results from vascular leakage following the local release of SP and CGRP during neurogenic inflammation (Fan et al., 2018). Similarly, acupuncture has been shown to induce the release of SP from the peripheral terminals of primary sensory neurons in rats (Kashiba and Uedo, 1991) and dogs (Chang et al., 1998). Based on these studies, we hypothesized that SP released under pathological conditions would cause physiological changes in acupoint tissue, enhance sensory afferent responses to the needling of acupoints, and thus play an important role in the generation of acupuncture effects.

We investigated whether: (1) cutaneous neurogenic inflammation and SP release at acupoints occurred as a result of the development of spontaneous hypertension; (2) stimulation of the acupoints alleviated hypertension and produced an increase of SP at acupoints; (3) SP enhanced the sensitivity of afferent nerves to acupuncture; and (4) SP or acupuncture increased the firing rates of wide dynamic range (WDR) neurons in the spinal cord and rostral ventrolateral medulla (rVLM) neurons in the midbrain.

\section{Materials and methods}

\subsection{Animals}

Adult male Sprague-Dawley rats (Hyochang, Seoul, Korea) weighing 250-350 g were used. Animals were housed at constant humidity $(40 \sim 60 \%)$ and temperature $\left(22 \pm 2{ }^{\circ} \mathrm{C}\right)$ with a 12-hour light/dark cycle and allowed free access to food and water. All procedures were carried out in accordance with the National Institutes of Health Guide for the Care and Use of Laboratory Animals and approved by the Institutional Animal Care and Use Committee (IACUC) at Daegu Haany University.

\subsection{Chemicals}

Evans blue dye (EBD; $50 \mathrm{mg} / \mathrm{ml}$ in saline; Sigma-Aldrich, USA), the SP receptor agonist substance $\mathrm{P}$ acetate salt hydrate (SP; $0.5 \mathrm{mg} / \mathrm{ml}$ in saline; $30 \mu \mathrm{L} /$ loci, subcutaneous (s.c.); Sigma-Aldrich), the SP receptor antagonist (+)-(2S, 3S)-3-(2-methoxybenzylamino)-2phenylpiperidine (CP-99994; $33 \mathrm{mmol} / \mathrm{ml}$ in saline; $30 \mu \mathrm{L} / \mathrm{loci}$, s.c.; TOCRIS, UK), were used (McLean et al., 1993). The transient receptor potential channel and vanilloid subfamily member 1 (TRPV1) agonist capsaicin (0.05\%; $20 \mu \mathrm{L} /$ loci, s.c.; Sigma-Aldrich), was dissolved in vehicle consisting of $10 \%$ alcohol and $10 \%$ Tween 80 in saline.

\subsection{Acupuncture treatment}

Acupuncture treatment was performed by using a mechanical acupuncture instrument (MAI) that was developed to mimic manual acupuncture stimulation. This device consisted of a custom-made control unit and a cell phone vibrator (MB-1203V, Motor Bank, Korea) 
attached to an acupuncture needle as described previously (Chang et al., 2017; Kim et al., 2013). While an assistant lightly restrained the rats, stainless steel needles (diameter 0.18 mm, length $8 \mathrm{~mm}$, Dongbang Acupuncture Inc., Qing Dao, China) were inserted bilaterally at a depth of $3 \mathrm{~mm}$ into neurogenic inflammatory spots, PC6 acupoints or the nearby points SI5 (located at the ulnar end of the transverse crease on the dorsal aspect of the wrist, $5 \mathrm{~mm}$ apart from PC6) and stimulated with the MAI for $20 \mathrm{sec}$ at $1 \mathrm{~min}$-intervals for a total of 10 min, unless stated otherwise.

\subsection{Immobilization stress-induced hypertension (IMH) and the measurement of systolic} blood pressure

Hypertension was induced by immobilization with a cone-shaped polyethylene bag, as described previously (Kim et al., 2017). Systolic blood pressure was measured noninvasively with a tail cuff blood pressure monitor (Model 47, IITC Inc., USA). Briefly, the rat was placed in a chamber, and an occluding cuff and a pneumatic pulse transducer were positioned on the base of the tail. A programmed electro-sphygmomanometer (Narco BioSystems Inc., USA) was inflated and deflated automatically, and the tail cuff signals from the transducer were automatically collected every 10 min using an IITC apparatus (Model 47, IITC Inc.). The mean of two readings was used for each blood pressure measurement.

\subsection{Detection of neurogenic inflammation in the skin by Evans blue dye injection}

Neurogenic inflammatory spots (neurogenic spots or Neuro-Sps) were visualized by intravenously injecting EBD $(50 \mathrm{mg} / \mathrm{kg})$ into male Sprague-Dawley rats as described previously (Kim et al., 2017). EBD was injected intravenously immediately after IMH induction. In detail, while the rat was immobilized by a cone-shaped bag, the distal portion of the tail was dipped into warm $40{ }^{\circ} \mathrm{C}$ water for at least $30 \mathrm{sec}$. EBD was then injected into the tail vein with a catheter (26 gauge), and skin color changes were observed up to $2 \mathrm{~h}$ after the injection. For an experiment of Supplementary Fig. 1, EBD was injected via jugular vein under isoflurane anesthesia. The blue-dyed areas on the skin were sketched using body charts, photographed with a digital camera (SONY ILCE-5000, China) and compared with a human acupoint chart based on the transpositional method, which locates acupoints on the surface of animal skin that correspond to the anatomical sites of human acupoints (Yin et al., 2008).

\subsection{Surgical injury of median and ulnar nerves}

Median and ulnar nerves were resected surgically as described previously (Fan et al., 2018). Briefly, under isoflurane anesthesia, a small skin incision was made longitudinally on the medial part of the elbow to expose the median and ulnar nerves. For surgical lesions of median and ulnar nerves, the nerves were bilaterally ligated with 4-0 silk and cut around the medial head of the triceps muscle of both forelimbs. The sham group underwent the same procedure but without nerve injury. Immediately after completion of surgery, the rats received intravenous EBD and IMH procedure. The numbers of Neuro-Sps following IMH induction were compared between nerve injury $(n=6)$ and sham $(n=6)$ groups. 


\subsection{Estimation of Evans blue dye extravasation in skin tissues}

The extent of EBD extravasation was assessed in the skin as described previously (Fan et al., 2018). After intravenous injection of EBD, the skin near the wrist was photographed using a digital camera (SONY ILCE-5000, China). The extent of the extravasation of EBD in the skin was estimated by using ImageJ software (National Institutes of Mental Health, USA) and expressed as a percentage of the basal blue pixel intensity (blue pixel value at the time point -basal blue pixel value before EBD)/basal blue pixel value before $\mathrm{EBD} \times 100$ ). The original EBD images were used to obtain a 3-dimensional (3D) surface plot with ImageJ software using the command Plugins/3D/interactive 3D surface plot (parameters: Mesh, Spectrum LUT, Scale 1.75, and Z 0.19). To further determine the concentration of EBD that infiltrated the tissues, the skin near the wrist was removed 120 min after EBD and processed using a dye extraction method as described previously (Martin et al., 2010). In brief, the skin (approximately $3 \mathrm{~mm}$ in size) near the wrist was excised, dry-weighed, homogenized in 1:3 vol of 50\% trichloroacetic acid (dissolved in $0.9 \%$ saline) and centrifuged (10,000 rpm for $10 \mathrm{~min})$. The supernatants were diluted with a $1: 3 \mathrm{vol}$ of $95 \%$ ethanol and measured using a spectrophotometric method ( $620 \mathrm{~nm}$ excitation/680 $\mathrm{nm}$ emission).

\subsection{In vivo fluorescence imaging of neurogenic inflammatory spots}

Animals were divided into the normal and IMH groups ( $\mathrm{n}=6$ per group). The rats were anesthetized $2 \mathrm{~h}$ after restraint and intravenous injection of EBD, and in vivo fluorescence signals were sequentially acquired with a $470 \mathrm{~nm}$ excitation filter and a $680 \mathrm{~nm}$ emission filter using bin 8 and $\mathrm{f} 2$ with the IVIS lumina III system (Perkin Elmer Company, USA).

\subsection{Immunohistochemical detection of substance $P$ and protein gene product (PGP) 9.5 in the skin}

Skin samples were taken from the wrists of IMH rats $(n=6)$ and naïve rats $(n=6)$, most of which commonly showed EBD leakage. In another set of animals $(n=6)$, MAI acupuncture was applied to the wrist skin. One hour after restraint or acupuncture, the samples were taken out, paraffin-embedded, sectioned $(5 \mu \mathrm{m})$ and then mounted on gelatin-coated slides. The slides were de-paraffinized with a series of different concentrations of xylene or ethanol and then rinsed under cold tap water. The slides were incubated with rabbit polyclonal SP antibody (1:400; Bioss, Woburn, USA; bs-0056R) and mouse monoclonal PGP 9.5 antibody (1:1000; Abcam, Cambridge, United Kingdom; ab8189), followed by incubation with secondary antibodies (1:200, Alexa Fluor® 488 donkey anti-rabbit IgG antibody, Thermo Scientific, MA, USA, Product\# A-21206; 1:200, Alexa Fluor® 594 donkey anti-mouse IgG antibody, Thermo Scientific, Product\# A-21203). The slides were washed and cover-slipped with Vectashield Hard Set mounting medium with DAPI (4', 6-diamidino-2-phenylindole; blue, Vector, USA, Product\# H-1500). Skin images were obtained from 3 sections from each animal with a laser-scanning confocal microscope (LSM800, Carl Zeiss, Germany) and quantified by using ImageJ software. Pixels with green fluorescence intensity greater than the cut-off value (100) were counted to quantify positive staining. Data were expressed as the number of positive pixels within a field area of $1280 \times 1024$ pixels. 


\subsection{Single fiber recordings of somatic afferent fibers}

Single fiber recordings of peripheral nerves were performed as described previously (Kim et al., 2013). The right median nerve was exposed between the medial belly of the triceps muscle and the humerus condyle and covered with mineral oil that was contained by skin flaps. The nerve was placed on a small mirror-based platform, and after the perineural sheath was removed, the nerve was teased into fine bundles and split to obtain a fine filament under a surgical microscope. The filament was looped around a silver-wire recording electrode, and the discharge activity was discriminated, recorded, and analyzed via a CED 1401 Micro3 device and Spike2 software (Cambridge Electronic Design, UK). To ensure that the measured unit activity was from the afferent fibers innervating PC6 acupoint over wrist, the action potential was evoked by mechanically stimulating PC6 acupoint over the wrist area using a von Frey filament (8.5 g force, Semmes-Weinstein Monofilaments; North Coast Medical, USA). The conduction velocity of the fibers was measured by dividing the conduction distance by the latency after the electrical stimulation artifact. The fiber types were classified according to their conduction velocity as described in previous studies (Harper and Lawson, 1985): $>2.2 \mathrm{~m} / \mathrm{sec}$ for A fibers and $<1.4 \mathrm{~m} / \mathrm{sec}$ for C fibers. Following injection of SP into PC6 acupoints, single fiber recordings from 15 afferent fibers $(9$ A- and $6 \mathrm{C}$-afferents) in 15 rats were carried out before and during acupuncture stimulation for 10 sec with the MAI.

\subsection{Extracellular recordings of wide dynamic range (WDR) neurons in the spinal cord}

Rats were anesthetized with an intraperitoneal injection of urethane $(1.5 \mathrm{~g} / \mathrm{kg})$. A laminectomy was performed to expose the spinal cord of C5-T1 level. The rat was placed in a stereotaxic apparatus, and the spinal cord was bathed in a pool of mineral oil. The body temperature was kept constant at $37{ }^{\circ} \mathrm{C}$ using a feedback-controlled DC heating pad. An extracellular recording was made for the WDR neurons in the dorsal horn, as described previously (Kim et al., 2015). Cells were recorded using a carbon filament glass microelectrode (0.4-1.2 M $\Omega$, Carbostar-1, Kation Scientific, USA) mounted on an electronic micromanipulator. Spontaneous discharges were amplified and filtered between $300 \mathrm{~Hz}$ and $10 \mathrm{kHz}$ (ISO-80; World Precision Instruments, USA). The single unit activity from the discharges was isolated, recorded and analyzed via a CED 1401 Micro3 device and Spike2 software (Cambridge Electronic Design). Deep WDR neurons were identified at recording depths of 500 to $1000 \mu \mathrm{m}$, according to the atlas compiled by Paxinos and Watson (Paxinos and Watson, 2007). Wide dynamic range (WDR) neurons were recognized based on their responses to both innocuous and noxious mechanical stimuli in a sensitive area near the PC6 or HT7 acupoints, most of which showed neurogenic inflammation in IMH rats. Brush stimuli and a set of von Frey filaments (Semmes-Weinstein Monofilaments; North Coast Medical, USA) were used to identify the dorsal horn neurons. WDR neurons with receptive fields located on the surface of the ipsilateral forepaw were recorded extracellularly. Specifically, as stimuli gradually progressed from non-noxious to noxious in intensity, each neuron that responded to the stimuli with a gradual increase in firing rate was classified as a WDR cell. After the receptive field of a WDR neuron was examined, graded mechanical stimuli were generated with non-noxious (2, 8.5 and $15 \mathrm{~g}$ forces) and noxious ( $60 \mathrm{~g}$ force) von Frey filaments (VFFs). All stimuli were applied at a rate of once per second for $10 \mathrm{sec}$ with a $20 \mathrm{sec}$ interval between stimuli. Background activity was recorded with VFFs with 2, 
8.5, 15 , and $60 \mathrm{~g}$ forces before the administration of treatment. The discharges of the WDR neurons were recorded $5 \mathrm{~min}, 10 \mathrm{~min}$, and $30 \mathrm{~min}$ after administration of saline, SP, SP receptor antagonist, and/or acupuncture treatment into receptive fields or Neuro-Sps. A total of 59 WDR neurons were recorded from 59 rats.

\subsection{In vivo extracellular single-unit recordings of rostral ventrolateral medulla (rVLM) neurons}

Single-unit discharges of rVLM neurons were recorded in anesthetized rats, as described previously (Shin et al., 2020). In brief, $1 \mathrm{~h}$ after immobilization with a cone-shaped polyethylene bag, rats were anesthetized with an intraperitoneal injection of urethane $(1.5 \mathrm{~g} /$ $\mathrm{kg})$. A carbon-filament glass microelectrode (0.4-1.2 M $\Omega$, Carbostar-1, Kation Scientific) was stereotaxically advanced to the rVLM (stereotaxic coordinates: posterior, $-11.96 \sim-$ $12.80 \mathrm{~mm}$; lateral, $1.9 \sim 2.4 \mathrm{~mm}$; deep, $9.8 \sim 10.6 \mathrm{~mm}$ ). Single-unit activity from the discharges was discriminated, recorded, and analyzed via a CED 1401 Micro3 device and Spike2 software (Cambridge Electronic Design). After recording stable baseline for at least 5 min, either saline ( $n=8), C P-99994(n=8 ; 30 \mu \mathrm{L}$, s.c.) or CAP was injected into NeuroSps near the PC6 acupoint area at the wrist and recordings were collected for $5 \mathrm{~min}$. Acupuncture stimulation with MAI at Neuro-Sps was then applied for 2 min to Neuro-Sps and the single-unit discharges were recorded for a further $5 \mathrm{~min}$. Additionally, the effects of intradermal injection of capsaicin (CAP; 0.05\%) into PC6 acupoint on single-unit discharges of rVLM neurons was tested in normal rats $(n=7)$. Upon completion of the recordings, the recording sites were marked by passing a $0.35 \mathrm{~mA}$ anodal current for $8 \mathrm{sec}$. The brains were removed, post-fixed, cryo-sectioned into $30 \mu \mathrm{m}$ thick sections and stained with toluidine blue and examined under a light microscope (N-200 M, China).

\subsection{Statistical analysis}

Statistical analysis was carried out using SigmaStat 3.5 software (Systat Software Inc., IL, USA). All data are presented as the mean \pm SEM (standard error of the mean) and analyzed by repeated measures of one or two-way analysis of variance (ANOVA) with Tukey post hoc tests or paired or unpaired $t$-tests, where appropriate. Statistical significance was denoted at $\mathrm{p}<0.05$.

\section{Results}

\subsection{Development of neurogenic inflammation at acupoints following Immobilization- induced hypertension}

First, we explored whether some acupoints displayed neurogenic inflammation in IMH rats.

After the initiation of restraint, the systolic blood pressure (BP) gradually increased over the next $2 \mathrm{~h}$ (two-way repeated ANOVA; group $\mathrm{F}_{(1,5)}=116.31, p<0.001$; time $\mathrm{F}_{(12,60)}=$ $16.725, p<0.001$; interaction $\mathrm{F}_{(12,60)}=15.879, p<0.001$; Fig. 1A, B) in IMH rats compared to naïve rats (Control, $\mathrm{n}=6$ ). Cutaneous neurogenic inflammatory sites (neurogenic spots or Neuro-Sps) began to appear, commonly at acupoint PC6, within $1 \mathrm{~min}$ after IMH induction. The color intensity at each of the acupoints gradually began to deepen and was fully saturated within $15 \mathrm{~min}$ after IMH and was significantly different when compared to the skin of naïve rats (two-way repeated ANOVA; group $\mathrm{F}_{(1,4)}=22.206, p=$ 
0.009; time $\mathrm{F}_{(17,68)}=5.836, p<0.001$; interaction $\mathrm{F}_{(17,68)}=6.092, p<0.001$; Fig. 1C, D). Neuro-Sps were found in the acupoints of the forelimb such as PC6 ( $n=9$ out of 12 sites) and HT7 $(n=8)$ and in the acupoints of the hind limb such as LR2 $(n=3), \operatorname{LR} 3(n=4)$, BL65 $(n=5)$ and BL66 $(n=4)$. In another set of animals, EBD was administered via jugular vein, but not tail vein, in IMH rats $(n=6)$. The Neuro-Sps were found in the forelimb and hind limb of IMH rats (in Supplementary Fig. 1) in a similar manner to that seen in tail vein injection. In vivo fluorescence imaging further confirmed the occurrence of the Neuro-Sps (Fig. 1E), suggesting that acupoints are most frequently located on the wrist and they exhibit the full development of cutaneous neurogenic inflammation within 15 min after IMH.

Further, these effects represent early-stage cutaneous changes in acupoint tissue resultant from IMH. To further test the involvement of axon reflex in the occurrence of Neuro-Sps, peripheral nerves (median/ulnar nerves) were cut to block the axon reflex as described previously (Willis et al., 2000), and the numbers of Neuro-Sps following IMH induction were compared between nerve injury $(n=6)$ and sham $(n=6)$ groups. The result showed that resection of median/ulnar nerves prior to IMH induction significantly reduced the number of Neuro-Sps compared to sham-operated IMH rats (Supplementary Fig. 2), suggesting the mediation of axon reflex by antidromic stimulation of sensory nerves.

\subsection{Increased substance $P$ in neurogenic spots}

To examine the increased release of SP from afferent nerves in Neuro-Sps, immunohistochemical detection of SP was performed on tissue samples from wrist areas of naïve (Control) and IMH rats (Fig. 2A, B). IMH rats (Hypertension, $n=6$ ) showed enhanced SP expression in Neuro-Sps compared to control rats $(n=6$; one-way repeated ANOVA, $\mathrm{F}_{(2,10)}=37.404, p<0.001$; Fig. 2B). Furthermore, the increase of SP in acupoints of IMH rats was not observed in non-acupoint areas (data not shown). To further determine whether acupuncture itself can increase SP release, MAI acupuncture stimulation was applied to the wrist area for $20 \mathrm{sec}$ at $1 \mathrm{~min}$-intervals for a total of $10 \mathrm{~min}$ in naïve rats $(n=6)$, revealing the enhanced expression of SP in the acupuncture-stimulated skin (Fig. 2C).

\subsection{Mediation by substance $P$ of the inhibitory effects of acupuncture on the development of hypertension}

To explore whether SP is involved in the effects of acupuncture on hypertension, we injected either vehicle (saline) or the SP receptor antagonist CP-99994 into Neuro-Sps over the wrist (mostly, PC6) 20 min prior to acupuncture in IMH rats. Acupuncture needles were inserted into bilateral Neuro-Sps and stimulated for $20 \mathrm{sec}$ at $1 \mathrm{~min}$-intervals for a total of $10 \mathrm{~min}$. Acupuncture at Neuro-Sps near the wrist, but not the nearby points SI5, prevented or reduced the elevation of blood pressure following restraint (Fig. 3A), which was prevented by pretreatment with CP-99994, but not vehicle at Neuro-Sps (two-way repeated ANOVA; group $\mathrm{F}_{(2,8)}=13.688, p=0.003$; time $\mathrm{F}_{(12,48)}=56.646, p<0.001$; interaction $\mathrm{F}_{(24,96)}=$ $1.768, p=0.028$; Fig. 3A). CP-99994 itself did not affect the development of hypertension (CP-99994 group in Fig. 3A). In another set of animals, the blockade of neurogenic inflammation at Neuro-Sps by CP-99994 was confirmed by 3D skin imaging (Fig. 3B) and measurement of the EBD concentration in the skin (one-way repeated ANOVA, $\mathrm{F}_{(2,13)}=$ $14.429, p<0.001$; Fig. 3C), indicating that the blockade of neurogenic inflammation by an 
SP receptor antagonist prior to acupuncture prevented the inhibitory effects of acupuncture on hypertension.

To determine whether an artificial increase in the SP level at acupoints generates acupuncture-like effects, subcutaneous injections of SP into Neuro-Sps were carried out in IMH rats. While IMH rats given vehicle developed hypertension, SP injection into NeuroSps near the wrist significantly inhibited the development of hypertension and mimicked

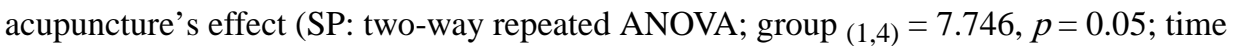
$\mathrm{F}_{(12,48)}=21.941, p<0.001 ;$ interaction ${ }_{(12,48)}=2.976, p=0.004 ;$ Fig. 3D).

It is known that capsaicin (CAP) induces the release of SP from primary afferent fibers (Purkiss et al., 2000). To further confirm that the elevation of SP levels in Neuro-Sps leads to the effects of acupuncture, we evaluated the effects of CP-99994 injections into Neuro-Sps. The subcutaneous injection of CAP mimicked the inhibitory effect of acupuncture or SP on the development of hypertension in IMH rats, which was prevented by pretreatment with CP-99994 (CAP: two-way repeated ANOVA; group $\mathrm{F}_{(1,4)}=34.336, p=0.004$; time $\mathrm{F}_{(12,48)}$ $=84.843, p<0.001$; interaction $\mathrm{F}_{(12,48)}=4.768, p<0.001$; Fig. 3G). In another set of experiments, we determined the levels of plasma extravasation $2 \mathrm{~h}$ after the injection of vehicle $(n=9)$ or SP $(n=9)$ in naïve and IMH rats and found significantly higher levels of plasma extravasation in wrist acupoints of SP-injected rats than in those of vehicle-injected rats, as shown in 3D skin images (Fig. 3E), and as assessed by spectrophotometry of EBD concentration ( $t$-test, $p<0.001$; Fig. 3F). These effects were replicated by the injection of CAP (Fig. 3H,I) into Neuro-Sps in naïve and IMH rats. Overall, these results suggest that the local release of SP in Neuro-Sps is closely linked to the effects of acupuncture.

\subsection{Enhancement by substance $\mathbf{P}$ of acupuncture-induced afferent fiber activation}

To determine whether SP increases the sensitivity of primary afferent fibers to acupuncture, single fiber recordings of peripheral nerves were performed in 15 somatic afferent neurons innervating the wrist area in 15 naïve rats (Fig. 4B). Among the 15 somatic afferents, 9 Aand $6 \mathrm{C}$-fibers were recorded. When the acupuncture needle was inserted into the wrist and mechanically stimulated with the MAI (Fig. 4A), the rats given intradermal SP into the receptive field over the wrist showed a 2-fold increase in A-fiber afferent discharges (70.89 $\pm 1.15 \mathrm{impulses} / \mathrm{sec})$ compared to the rats in the vehicle group $(33.33 \pm 0.71 \mathrm{impulses} / \mathrm{sec} ; t$ test, $p<0.001$; Fig. 4C1-3). The activity of C-fibers during acupuncture stimulation was also examined following intradermal SP injection. The intradermal injection of SP increased C-fiber afferent discharges by almost 3 -fold compared to vehicle group ( $7.93 \pm 0.26$ vs. $21.72 \pm 0.79$ impulses/sec; $t$-test, $p<0.001 ;$ Fig. 4D1-3), suggesting that the increased levels of SP enhanced the sensitivity of both somatic A- and C-afferents to acupuncture stimulation and significantly enhanced acupuncture signaling.

\subsection{Substance P-induced enhancement of spinal WDR neuron activity}

Next, we explored whether the activity of spinal dorsal horn neurons was enhanced by intradermal SP injection in normal rats and reversed by the subcutaneous injection of an SP receptor antagonist, CP-99994, into neurogenic acupoints in IMH rats. In addition, we investigated whether the activity of spinal dorsal horn neurons was activated by acupuncture 
treatment in normal rats and this effect was blocked by the subcutaneous injection of CP-99994 into the receptive field over the wrist. The responses of dorsal horn WDR neurons to mechanical stimuli were compared after injection with either saline $(n=11)$ or SP $(n=9)$ in normal rats. Neurons in the spinal dorsal horn responded well to a variety of mechanical stimuli, including the stroking of the skin with a brush and repeated applications of von Frey filaments (VFFs) with non-noxious (2, 8.5, and $15 \mathrm{~g}$ ) bending forces and noxious (60 g) bending forces. All recorded WDR neurons responded to progressively increasing VFF stimulation (bending forces of 2, 8.5, 15, and $60 \mathrm{~g}$ ) applied to the receptive fields. Representative examples are shown in Fig. 5B-I. In the saline group, the mean activity of the WDR neurons was $10.45 \pm 1.20,10.72 \pm 1.59,10.46 \pm 1.60$, and $10.97 \pm 1.66$ spikes/sec in response to a $60 \mathrm{~g}$ VFF-induced bending force at before, $5 \mathrm{~min}, 10 \mathrm{~min}$ and $30 \mathrm{~min}$, respectively. On the other hand, intradermal SP injection resulted in significantly increased evoked activity in response to a $60 \mathrm{~g}$ bending force (5 min, 19.16 $\pm 2.03 ; 10 \mathrm{~min}, 20.96 \pm$ $2.62 ; 30 \mathrm{~min}, 22.29 \pm 2.09 \mathrm{spikes} / \mathrm{sec}$, respectively) compared to that observed in the saline group (one-way repeated ANOVA, $5 \mathrm{~min}: \mathrm{F}(1,8)=7.942, p=0.023 ; 10$ min: $\mathrm{F}_{(1,8)}=24.551$, $p=0.001 ; 30$ min: $\mathrm{F}_{(1,8)}=20.332, p=0.002 ;$ Fig. $\left.5 \mathrm{~B}, \mathrm{C}, \mathrm{J}\right)$. However, there were no differences in the WDR discharge rates in response to bending forces of 2, 8.5 and $15 \mathrm{~g}$ between the saline and SP groups (Supplementary Fig. 3). This suggests that SP increased the mechanical sensitivity to noxious stimulation, but not non-noxious stimulation.

When the activity of WDR neurons was compared between subcutaneous injection of saline and CP-99994 into the Neuro-Sps in IMH rats, the injection of the SP receptor antagonist CP-99994 into Neuro-Sps (PC6 acupoint area) in IMH rats reduced the level of WDR discharges compared to saline treatment (one-way repeated ANOVA, 5 min: $\mathrm{F}_{(1,5)}=8.804, p$ $=0.031 ; 10$ min: $\mathrm{F}_{(1,5)}=8.689, p=0.032 ; 30$ min: $\mathrm{F}_{(1,5)}=11.536, p=0.019$; Fig. 5D, E, J). The mean activity of WDR neurons was $21.96 \pm 0.25,9.78 \pm 0.71,7.46 \pm 0.35$, and $7.65 \pm$ $0.78 \mathrm{spikes} / \mathrm{sec}$ in response to $60 \mathrm{~g}$ of von Frey filament bending force at before and $5 \mathrm{~min}$, $10 \mathrm{~min}$ and $30 \mathrm{~min}$ after the injection of an SP receptor antagonist, respectively. This result suggests that blockade of SP by CP-99994 at Neuro-Sps inhibited the WDR activities in IMH rats.

Next, to determine if the evoked activity of WDR neurons was activated by acupuncture stimulation and blocked by an intradermal CP-99994 injection. We tested the effects of subcutaneous injection of CP-99994, which resulted in significantly decreased evoked activity in response to a $60 \mathrm{~g}$ bending force ( $5 \mathrm{~min}, 12.63 \pm 1.88 ; 10 \mathrm{~min}, 11.84 \pm 1.19 ; 30$ min, $13.03 \pm 1.28$ spikes/sec, respectively) compared to that observed in the saline-Acup group (one-way repeated ANOVA, 5 min: $\mathrm{F}_{(1,5)}=38.288, p=0.002 ; 10 \mathrm{~min}: \mathrm{F}_{(1,5)}=8.934$, $p=0.03 ; 30$ min: $\mathrm{F}_{(1,5)}=9.868, p=0.026$; Fig. $\left.5 \mathrm{~F}, \mathrm{G}, \mathrm{J}\right)$. Moreover, the evoked activity of WDR neurons was enhanced by acupuncture stimulation, which was suppressed by CP-99994 in IMH rats (60 $\mathrm{g}$ bending force; one-way repeated ANOVA, 5 min: $\mathrm{F}_{(1,5)}=$ 9.100, $p=0.03$; 10 min: $\mathrm{F}_{(1,5)}=7.814, p=0.038 ; 30$ min: $\mathrm{F}_{(1,5)}=9.923, p=0.025$; Fig. 5H, I, J). This indicates that acupuncture effects on the activity of WDR neurons are associated with SP. Overall, these data indicate that increased SP release in acupoints enhanced mechanical sensitivity to noxious stimuli such as needles. 


\subsection{Inhibition by substance $P$ receptor antagonist of acupuncture-induced rVLM neuronal activation}

Finally, to determine whether acupuncture at Neuro-Sps excites rVLM neurons, known as an important site for cardiovascular regulation (Sved et al., 2003), and if acupuncture effects on rVLM neurons might be inhibited by subcutaneous injection of an SP receptor antagonist in Neuro-Sps prior to acupuncture, we performed in vivo extracellular recording of rVLM neurons and tested the effects of Neuro-Sp injection of CP-99994 on rVLM excitability. When acupuncture stimulation at Neuro-Sps near the wrist was applied for 2 min, singleunit discharges increased up to about $130 \%$ over baseline and returned to baseline $2 \mathrm{~min}$ after stimulation (Fig. 6A). On the other hand, acupuncture treatment after subcutaneous injection of CP-99994 into Neuro-Sps prevented the increase of the firing rates of rVLM neurons following acupuncture stimulation (Fig. 6B), compared to that of MAI treatment after saline injection (in IMH rat, one-way repeated ANOVA, $\mathrm{F}_{(2,14)}=23.382, p<0.05$; Fig. $6 C)$.

To further confirm that the elevation of subcutaneous SP levels in Neuro-Sps is associated with the rVLM activation following acupuncture, we injected CAP into Neuro-Sps to induce SP release and measured the excitability of rVLM neurons. The subcutaneous injection of CAP mimicked the excitatory effect of acupuncture in naïve rats ( $t$-test, $p<0.05$; Fig. 6D, E), suggesting that the increased levels of SP in acupoints contributed to enhanced firing activities of rVLM and generated acupuncture effects in IMH rats.

\section{Discussion}

Acupoints near the wrist showed increased neurogenic inflammation following the development of hypertension. The Neuro-Sps showed increased SP release from the afferent nerve. The stimulation of Neuro-Sps inhibited the development of hypertension, which was reversed by the local injection of an SP receptor antagonist into Neuro-Sps prior to acupuncture. These acupuncture effects were also mimicked by the direct injection of either SP or CAP into the Neuro-Sps. Single fiber recording of peripheral nerve fibers revealed that the local injection of SP into Neuro-Sps increased the sensitivity of A- and C-fibers in response to acupuncture stimulation. The discharge rates of spinal WDR neurons to VFFs stimulation of Neuro-Sps were increased in IMH rats compared to those in normal rats, which was blocked by the injection of the SP receptor antagonist CP-99994. The WDR activity was elevated following intradermal SP treatment or acupuncture stimulation in naïve or IMH rats. In addition, stimulation of Neuro-Sps increased rVLM neuronal activity in the midbrain, which was blocked by injection CP-99994 into Neuro-Sps prior to acupuncture and mimicked by the local injection of CAP, suggesting that elevated SP levels in acupoints critically contribute to the triggering of acupuncture signals and the generation of acupuncture effects.

Our previous studies showed that in rat models of hypertension or colitis, the skin over acupoints exhibits neurogenic inflammation due to the viscerosomatic convergence of sensory pathways suggesting that acupoints appear to be identical to cutaneous neurogenic inflammation spots (Neuro-Sps) on the skin (Kim et al., 2017, 2006). Consistent with our previous study (Fan et al., 2018), the present study showed that cutaneous neurogenic 
inflammation manifested in acupoints, such as PC6 on the wrist in IMH rats, a common treatment site for cardiac disorders ( $\mathrm{Li}$ et al., 2012) or others that have been shown to be effective for cardiovascular disorders (He et al., 2017; Longhurst and Tjen, 2013).

Consistent with our previous study (Kim et al., 2017), Neuro-Sps were observed most frequently in the acupoints of distal extremities and also found in other areas such as thigh and lower back. High prevalence of the spots on distal extremities may be due to high density of sensory innervation in distal extremities (Blake et al., 2002). It has been proposed that acupoints have a dynamic nature; their appearance varies in response to individual pathological conditions (Dung, 2013). It has been unclear how quickly acupuncture points appear under pathological conditions. In the present study, neurogenic inflammation began to appear in acupoints within $1 \mathrm{~min}$ after the IMH procedure and had fully developed within 15 min and was maintained during IMH. This suggests that the topical changes in acupoints preceded the onset of pathological conditions and were maintained during the pathological condition.

The small diameter nociceptive afferents can be classified as the peptidergic and the nonpeptidergic (Alvarez and Fyffe, 2000). As the peptidergic afferents possess the neuropeptides SP and CGRP, activation of the peptidergic induces the release of SP and CGRP into the periphery (Richardson and Vasko, 2002; Schmelz and Petersen, 2001). These neuropeptides play a role in neurogenic inflammation in the skin by activating vasodilation, axon reflex flare, and microvascular plasma extravasation (Schmelz and Petersen, 2001; Wesselmann and Lai, 1997). In the present study, Neuro-Sps over the wrist showed significant increases in SP levels in epidermis and dermis, involving areas expressing PGP 9.5 (a nerve terminal marker) in IMH rats, suggesting that primary afferent fibers are activated during the development of hypertension, which induces the release of SP from the activated afferent fibers in Neuro-Sps. Moreover, peripheral sympathetic function appears to be increased during the development of immobilization-induced hypertension (Lamprecht et al., 1973; Nankova et al., 1996). The interaction between primary afferent neurons and sympathetic neurons can take place proximal and/or distal to the neurovascular junction and is responsible for sympathetic modulation of neurogenic inflammation (Häbler, 2007). Between the development of hypertension and SP release in Neuro-Sps, the sympathetic nervous system may also be involved. Consistent with the present data (Fig. 2), previous studies reported that acupuncture induced the release of SP from the peripheral terminals of primary sensory neurons in rats (Kashiba and Uedo, 1991) and dogs (Chang et al., 1998). However, little is known about the relationship between SP in acupoints and the effects of acupuncture. In the present study, acupuncture produced inhibitory effects on the development of hypertension, and these effects were replicated by the intradermal injection of SP into Neuro-Sps. In contrast, the pretreatment of Neuro-Sps with an SP receptor antagonist prior to acupuncture abolished the effects of acupuncture on hypertension. The present data further confirmed that the injection of CAP, which is known to release SP from afferent fibers (Purkiss et al., 2000), into Neuro-Sps prevented the development of hypertension. These results suggest that SP is an important neuropeptide for the generation of acupuncture effects.

In somatic areas affected by referred pain from viscera, Neuro-Sps are generated by the activation of small diameter sensory afferents (C/A $\delta$-fibers) in the dermatome that are 
convergent with visceral afferents (Arendt-Nielsen et al., 2008; Wesselmann and Lai, 1997). Sensory neurons are branched, with one projection leading to the internal organs and the other extending to the skin. Our previous study found convergent dorsal root ganglion neurons that innervated both the heart and Neuro-Sps (Kim et al., 2017). The visceral inputs activate the viscerosomatic convergent neurons in the sensory pathway, and the neurons antidromically activate the branches, leading to the release of SP from small diameter sensory fibers and subsequent neurogenic extravasation (Arendt-Nielsen et al., 2008; Wesselmann and Lai, 1997) and sensitization of primary afferent nociceptors (Sahbaie et al., 2009). In the present study, neurogenic inflammation in acupoints appeared at the beginning of and continued until the end of the development period of hypertension in IMH rats, and the Neuro-Sps showed increased SP release. Furthermore, our single fiber recordings showed increased needle sensitivity in the Neuro-Sps following pretreatment with SP. In our in vivo extracellular recordings, the evoked responses of WDR neurons in the spinal dorsal horn were greatly enhanced by the injection of SP into Neuro-Sps in normal rats or immobilization-induced hypertension (IMH) rats, which was reversed by the injection of CP-99994 (SP receptor antagonist) in the IMH rat model. It has been suggested that active acupoints are associated with tissues where the sensory nerve endings are sensitized by neurogenic inflammatory mediators (He et al., 2017; Rong et al., 2013). Given that the sensitized sensory nerve endings are more sensitive to external stimuli than intact sensory nerves, we suggest that sensory nerve endings in acupoints would be sensitized by SP released during neurogenic inflammation in IMH rats. Accordingly, the stimulation of these sensitive acupoints would evoke the therapeutic effects of acupuncture by reaching physiological thresholds more easily when comparable to those associated with the stimulation of normal surrounding tissues, including sham or inactive acupoints.

Intradermal SP enhanced single fiber activity and the excitability of spinal WDR neurons in response to noxious mechanical stimulation. Moreover, the excitability of spinal WDR neurons induced by the stimulation of Neuro-Sps was reversed by the subcutaneous injection of the SP receptor antagonist prior to acupuncture. This suggests that elevated SP primarily sensitizes acupoints at the level of the dermatome, not the spinal level, and the SP in turn stimulates the onset of acupuncture effects, and its action is largely dependent on the cutaneous SP level. However, it is not clear how SP induces acupuncture effects in the skin. As one possible explanation for the role of SP in acupuncture effects, the interaction of SP and NK1R in keratinocytes may be considered. The released SP triggers a number of biological responses by stimulating the tachykinin NK1R. SP has been shown to regulate the production of cytokines, including IL-1 $\beta$, IL-6 and TNF-a (Dallos et al., 2006), in skin keratinocytes. As these cytokines have also been linked to acupuncture effects (Zhang et al., 2012), we suggest that stimulation with acupuncture needles induces the release of SP from sensory afferent terminals and in turn generates cytokines by activating NK1 receptors in keratinocytes, which induces acupuncture effects. Another possible explanation is the interaction of SP and mast cells. A previous study suggested that acupuncture increases the levels of neuropeptides such as SP in subepidermal nerve fibers and activates mast cells. The mast cells aggregate and degranulate, which is accompanied by the release of tryptase and serotonin, and this may contribute to the initiation of acupuncture signals to generate the effects of acupuncture (Chen et al., 2018). 
Intradermal SP induced a 2-fold increase in afferent A-fiber activity and a 3-fold increase in afferent $\mathrm{C}$-fiber activity, respectively, compared to those of control groups, indicating that intradermal SP increased both A- and C-fiber activities to the similar extent. Although the most convincing evidence for a role of SP comes from studies on the $\mathrm{C}$ fibers, it is also known that 20-30\% of A $\delta$-fibers contain SP (Lawson et al., 1993; McCarthy and Lawson, 1989). Unfortunately, there are no studies directly investigating the effect of intradermal SP on primary afferent A- and C-fiber activity. In one study using co-administration of glutamate and SP, the glutamate-induced responses of primary afferent A $\delta$-or C-fiber activities were increased up to about 3-fold by intradermal SP (Zhang et al., 2006). Thus, it is likely that increase of A-fiber activity following intradermal SP in the present study is due to activation of $A \delta$ fiber via interaction of cutaneous NK1R-SP.

The rVLM plays an important role in regulating sympathetic activity associated with cardiovascular function (Sved et al., 2003) and stimulation of the PC6 acupoint significantly attenuates visceral sympathoexcitatory reflex responses through activation of opioid receptors in the rVLM (Chao et al., 1999; Li et al., 2002; Zhou et al., 2005a). Consistent with previous studies (Shin et al., 2020; Zhou et al., 2005b), the present study revealed that stimulation of Neuro-Sps near the wrist increased rVLM neuronal activity in IMH rats, which was prevented by local injection of SP receptor antagonist into Neuro-Sps. This suggests that locally released SP is linked with rVLM neuronal activity following acupuncture.

\section{Conclusions}

In conclusion, our findings suggest that SP released during neurogenic inflammation enhances the responses of sensory afferents to the needling of acupoints and serves as an important neuropeptide involved in the initiation of acupuncture effects.

\section{Supplementary Material}

Refer to Web version on PubMed Central for supplementary material.

\section{Acknowledgements}

This research was supported by a National Research Foundation of Korea (NRF) grant funded by the Korea government (MSIT) (No. 2018R1A5A2025272, 2019R1A2C1002555), the Korea Institute of Oriental Medicine (KIOM) (KSN1812181, KSN2013210), and USPHS NIH grant AT009945.

\section{Abbreviations:}

$\begin{array}{ll}\text { Acup } & \text { Acupuncture } \\ \text { BP } & \text { Blood pressure } \\ \text { CGRP } & \text { Calcitonin gene-related peptide } \\ \text { EBD } & \text { Evans blue dye } \\ \text { IMH } & \text { Immobilization stress-induced hypertension }\end{array}$




$\begin{array}{ll}\text { MAI } & \text { Mechanical acupuncture instrument } \\ \text { Neuro-Sps } & \text { Neurogenic spots or neurogenic inflammatory spots } \\ \text { NK1R } & \text { Neurokinin 1 receptor } \\ \text { PGP } & \text { Protein gene product } \\ \text { rVLM } & \text { Rostral ventrolateral medulla } \\ \text { SP } & \text { Substance P } \\ \text { TRPV1 } & \text { Transient receptor potential channel, vanilloid subfamily member 1 } \\ \text { VFFs } & \text { von Frey filaments } \\ \text { WDR } & \text { Wide dynamic range }\end{array}$

\section{References}

Alvarez FJ, Fyffe REW, 2000 Nociceptors for the 21st Century. Curr. Rev. Pain 4 (6),451-458. 10.1007/s11916-000-0069-4. [PubMed: 11060591]

Arendt-Nielsen L, Schipper KP, Dimcevski G, Sumikura H, Krarup AL,Giamberardino MA, Drewes AM, 2008 Viscero-somatic reflexes in referred pain areas evoked by capsaicin stimulation of the human gut. Eur. J. Pain (London, England) 12, 544-551. doi: 10.1016/j.ejpain.2007.08.010.

Blake DT, Byl NN, Merzenich MM, 2002 Representation of the hand in the cerebral cortex. Behav. Brain Res 135 (1-2), 179-184. 10.1016/s0166-4328(02)00163-8. [PubMed: 12356448]

Chang WW, Weissensteiner H, Rausch WD, Chen KY, Wu LS, Lin JH, 1998 Comparison of substance $\mathrm{P}$ concentration in acupuncture points in different tissues in dogs. Am. J. Chin. Med 26 (01), 13-18. 10.1142/s0192415x98000038. [PubMed: 9592589]

Chang S, Ryu Y, Gwak YS, Kim NJ, Kim JM, Lee JY, Kim SA, Lee BH,Steffensen SC, Jang EY, Yang $\mathrm{CH}$, Kim HY, 2017 Spinal pathways involved in somatosensory inhibition of the psychomotor actions of cocaine. Sci. Rep 7 (1). 10.1038/s41598-017-05681-7.

Chao DM, Shen LL, Tjen-A-Looi S, Pitsillides KF, Li P, Longhurst JC, 1999 Naloxone reverses inhibitory effect of electroacupuncture on sympathetic cardiovascular reflex responses. Am. J. Physiol.-Heart Circulatory Physiol 276 (6), H2127-H2134.

Chen L-Z, Kan Y.u., Zhang Z-Y, Wang Y-L, Zhang X-N, Wang X-Y, He W, Jing X-H, 2018 Neuropeptide initiated mast cell activation by transcutaneous electrical acupoint stimulation of acupoint LI4 in rats. Sci. Rep 8 (1). 10.1038/s41598-018-32048-3.

Corrigan F, Vink R, Turner RJ, 2016 Inflammation in acute CNS injury: a focus on the role of substance P: neurogenic inflammation in acute CNS injury. Br. J. Pharmacol 173 (4), 703-715. 10.1111/bph.13155. [PubMed: 25827155]

Dallos A, Kiss M, Polyánka H, Dobozy A, Kemény L, Husz S, 2006 Effects of the neuropeptides substance $\mathrm{P}$, calcitonin gene-related peptide, vasoactive intestinal polypeptide and galanin on the production of nerve growth factor and inflammatory cytokines in cultured human keratinocytes. Neuropeptides 40 (4), 251-263. 10.1016/j.npep.2006.06.002. [PubMed: 16904178]

Douglas SD, Leeman SE, 2011 Neurokinin-1 receptor: functional significance in the immune system in reference to selected infections and inflammation. Annals of the New York Academy of Sciences 1217, 83-95. doi: 10.1111/j.1749-6632.2010.05826.x. [PubMed: 21091716]

Dung H, 2013 Acupuncture: An Anatomical Approach. Routledge.

Fan Y, Kim DH, Ryu Y, Chang S, Lee BH, Yang CH, Kim HY, 2018 Neuropeptides SP and CGRP Underlie the Electrical Properties of Acupoints. Front Neurosci 12, 907. doi: 10.3389/ fnins.2018.00907. [PubMed: 30618546]

Garcia-Recio S, Gascón P, 2015 Biological and pharmacological aspects of the NK1-receptor. Biomed Res. Int 2015, 1-14. 10.1155/2015/495704. 
Häbler H-J, 2007 Neurogenic Inflammation and Sympathetic Nervous System In: Schmidt RF, Willis WD (Eds.), Encyclopedia of Pain. Springer Berlin Heidelberg, Berlin, Heidelberg, pp. 1276-1278.

Harper AA, Lawson SN, 1985 Conduction velocity is related to morphological cell type in rat dorsal root ganglion neurones. J. Physiol, 359, 31-46. [PubMed: 3999040]

He W, Wang X-Y, Shi H, Bai W-Z, Cheng B, Su Y-S, Yu X-C, Jing X-H, Zhu B, 2017 Cutaneous neurogenic inflammation in the sensitized acupoints induced by gastric mucosal injury in rats. BMC Complement. Altern. Med 17 (1). 10.1186/s12906-017-1580-z.

Huang S-C, Korlipara VL, 2010 Neurokinin-1 receptor antagonists: a comprehensive patent survey. Expert Opin. Ther. Pat 20 (8), 1019-1045. [PubMed: 20533894]

Kashiba H, Uedo Y, 1991 Acupuncture to the skin induces release of substance p and calcitonin generelated peptide from peripheral terminals of primary sensory neurons in the rat. Am. J. Chin. Med 19, 189-197. [PubMed: 1722639]

Kim D-H, Ryu Y, Hahm DH, Sohn BY, Shim I, Kwon OS, Chang S, Gwak YS, Kim MS, Kim JH, Lee BH, Jang EY, Zhao R, Chung JM, Yang CH, Kim HY, 2017 Acupuncture points can be identified as cutaneous neurogenic inflammatory spots. Sci. Rep 7 (1). 10.1038/s41598-017-14359-Z.

Kim H-Y, Hahm D-H, Sohn B-Y, Choi Y, Pyun K-H, Lee H-J, Shim I, 2006 Skin on GV01 acupoint in colonic inflammatory states: tenderness and neurogenic inflammation. J. Physiol. Sci 56 (4), 317 320. 10.2170/physiolsci.SC001606. [PubMed: 16911811]

Kim HY, Lee I, Chun SW, Kim HK, 2015 Reactive oxygen species donors increase the responsiveness of dorsal horn neurons and induce mechanical hyperalgesia in rats. Neural Plasticity 2015, 1-10. $10.1155 / 2015 / 293423$.

Kim SA, Lee BH, Bae JH, Kim KJ, Steffensen SC, Ryu YH, Leem JW, Yang CH, Kim HY, 2013 Peripheral afferent mechanisms underlying acupuncture inhibition of cocaine behavioral effects in rats. PloS one 8, e81018. doi: 10.1371/journal.pone.0081018. [PubMed: 24260531]

Lamprecht F, Williams RB, Kopin IJ, 1973 Serum dopamine-beta-hydroxylase during development of immobilization-induced hypertension. Endocrinology 92, 953-956. doi: 10.1210/endo-92-3-953. [PubMed: 4701493]

Lawson SN, Perry MJ, Prabhakar E, McCarthy PW, 1993 Primary sensory neurones: Neurofilament, neuropeptides and conduction velocity. Brain Res. Bull 30 (3-4), 239-243. 10.1016/0361-9230(93)90250-f. [PubMed: 7681350]

Li J, Li J, Chen Z, Liang F, Wu S, Wang H, 2012 The influence of pc6 on cardiovascular disorders: a review of central neural mechanisms. Acupunct. Med 30 (1), 47-50. [PubMed: 22378585]

Li P, Rowshan K, Crisostomo M, Tjen-A-Looi SC, Longhurst JC, 2002 Effect of electroacupuncture on pressor reflex during gastric distension. Am. J. Physiol.-Regulatory, Integrative Comparative Physiol 283 (6), R1335-R1345.

Longhurst JC, Tjen ALS, 2013 Acupuncture regulation of blood pressure: two decades of research. Int. Rev. Neurobiol 111, 257-271. doi: 10.1016/b978-0-12-411545-3.00013-4. [PubMed: 24215927]

Mantyh PW, 2002 Neurobiology of substance P and the NK1 receptor. J. Clin.Psychiatry 63 (Suppl. 11), 6-10. [PubMed: 12562137]

Martin Y, Avendaño C, Piedras MJ, Krzyzanowska A, 2010 Evaluation of Evans Blue extravasation as a measure of peripheral inflammation. Protocol Exchange 10, 1919-1931. doi: 10.1038/ protex.2010.209.

Martinez AN, Philipp MT, 2016 Substance P and antagonists of the neurokinin-1 receptor in neuroinflammation associated with infectious and neurodegenerative diseases of the central nervous system. J. Neurol. Neuromed 1, 29-36.

McCarthy PW, Lawson SN, 1989 Cell type and conduction velocity of rat primary sensory neurons with substance p-like immunoreactivity. Neuroscience 28 (3), 745-753. [PubMed: 2469036]

McLean S, Ganong A, Seymour PA, Snider RM, Desai MC, Rosen T, Bryce DK, Longo KP, Reynolds LS, Robinson G, et al., 1993 Pharmacology of CP-99,994; a nonpeptide antagonist of the tachykinin neurokinin-1 receptor. J. Pharmacol. Experimental Therapeutics 267, 472-479.

Nankova B, Kvetnansky R, Hiremagalur B, Sabban B, Rusnak M, Sabban EL, 1996 Immobilization stress elevates gene expression for catecholamine biosynthetic enzymes and some neuropeptides in rat sympathetic ganglia: effects of adrenocorticotropin and glucocorticoids. Endocrinology 137 (12), 5597-5604. [PubMed: 8940389] 
Paxinos G, Watson C, 2007 The Rat Brain in Stereotaxic Coordinates in Stereotaxic Coordinates. Elsevier.

Purkiss J, Welch M, Doward S, Foster K, 2000 Capsaicin-stimulated release of substance P from cultured dorsal root ganglion neurons: involvement of two distinct mechanisms. Biochem. Pharmacol 59 (11), 1403-1406. [PubMed: 10751549]

Richardson JD, Vasko MR, 2002 Cellular mechanisms of neurogenic inflammation. J.Pharmacol. Exp. Ther 302 (3), 839-845. [PubMed: 12183638]

Rong P, Zhu B, Li Y, Gao X, Ben H, Li Y, Li L, He W, Liu R, Yu L, 2011 Mechanism of acupuncture regulating visceral sensation and mobility. Front. Med 5 (2), 151-156. 10.1007/ s11684-011-0129-7. [PubMed: 21695619]

Rong P-J, Li S, Ben H, Li L, Yu L-L, Cui C-X, Li X, Zhu B, 2013 Peripheral and spinal mechanisms of acupoint sensitization phenomenon. Evidence-Based Complementary and Alternative Medicine 2013, 1-6. 10.1155/2013/742195.

Sahbaie P, Shi X, Guo T-Z, Qiao Y, Yeomans DC, Kingery WS, Clark DJ, 2009 Role of substance P signaling in enhanced nociceptive sensitization and local cytokine production after incision: Pain 145 (3), 341-349. 10.1016/j.pain.2009.06.037. [PubMed: 19660865]

Schmelz M, Petersen LJ, 2001 Neurogenic inflammation in human and rodent skin. Physiology 16 (1), 33-37.

Shin JH, Fan Y.u., Kim D-H, Jang HB, Chang S, Ryu Y, Bae JH, Lee S, Lee BH, Steffensen SC, Yang $\mathrm{CH}$, Kim HY, 2020 Paired mechanical and electrical acupuncture of neurogenic spots induces opioid-mediated suppression of hypertension in rats. J. Physiol. Sci 70 (1). 10.1186/ s12576-020-00735-4.

Stux G, Pomeranz B, 1987 Acupuncture: Textbook and Atlas. Springer-Verlag Berlin Heidelberg. doi: 10.1007/978-3-642-71742-0.

Sved A, Ito S, Sved J, 2003 Brainstem mechanisms of hypertension: Role of the rostral ventrolateral medulla. Current Hypertension Reports 5, 262-268. doi: 10.1007/s11906-003-0030-0. [PubMed: 12724060]

Wesselmann U, Lai J, 1997 Mechanisms of referred visceral pain: uterine inflammation in the adult virgin rat results in neurogenic plasma extravasation in the skin. Pain 73 (3), 309-317. [PubMed: 9469520]

Willis WD, Wu J, Lin Q, 2000 The Role of Dorsal Root Reflexes in Neurogenic Inflammation and Pain In: Saadé NE, Apkarian AV, Jabbur SJ (Eds.), Pain and Neuroimmune Interactions. Springer US, Boston, MA, pp. 99-110.

Yin CS, Jeong H-S, Park H-J, Baik Y, Yoon M-H, Choi C-B, Koh HG, 2008 A proposed transpositional acupoint system in a mouse and rat model. Res. Vet. Sci 84(2), 159-165. 10.1016/ j.rvsc.2007.04.004. [PubMed: 17559895]

Zhang Q, Zhao Y, Guo Y, Cao DY, Tian YL, Yao FR, Wang HS, 2006 Electrophysiological evidence for the interaction of substance $\mathrm{P}$ and glutamate on Adelta and $\mathrm{C}$ afferent fibre activity in rat hairy skin. Clin. Exp. Pharmacol. Physiol 33, 1128-1133. doi: 10.1111/j.1440-1681.2006.04504.x. [PubMed: 17184490]

Zhang Z-J, Wang X-M, McAlonan GM, 2012 Neural acupuncture unit: a new concept for interpreting effects and mechanisms of acupuncture. Evidence-Based Complementary and Alternative Medicine 2012, 1-23. 10.1155/2012/429412.

Zhou W, Fu L-W, Tjen-A-Looi SC, Li P, Longhurst JC, 2005a Afferent mechanisms underlying stimulation modality-related modulation of acupuncture-related cardiovascular responses. J. Appl. Physiol 98 (3), 872-880. 10.1152/japplphysiol.01079.2004. [PubMed: 15531558]

Zhou W, Tjen-A-Looi SC, Longhurst JC, 2005b Brain stem mechanisms underlying acupuncture modality-related modulation of cardiovascular responses in rats. J. Appl. Physiol 99 (3), 851-860. 10.1152/japplphysiol.01365.2004. [PubMed: 15817715] 
A

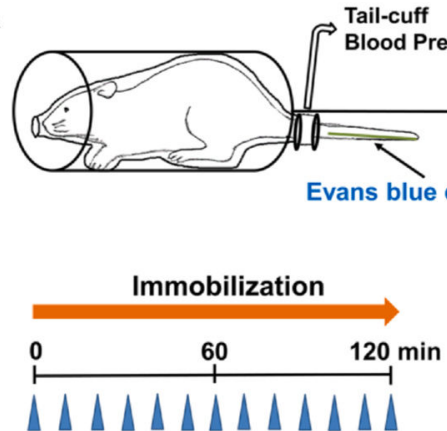

Measurement of Blood Pressure

C

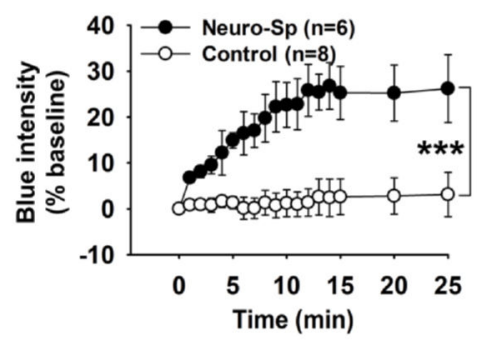

D

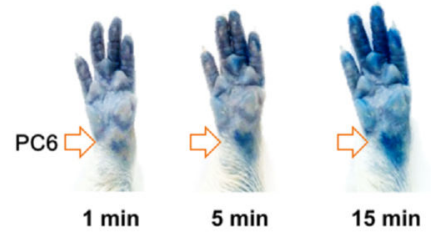

B

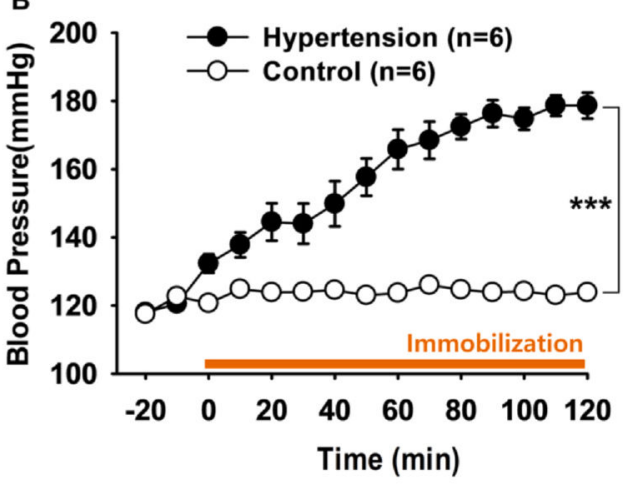

E

Control
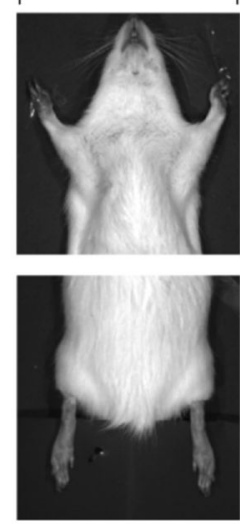

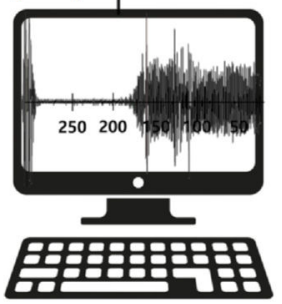

Hypertension

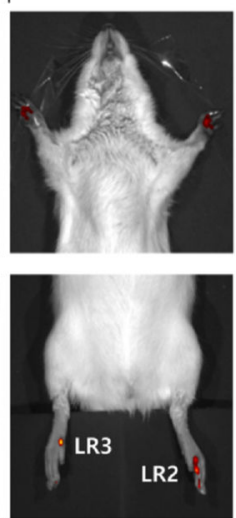

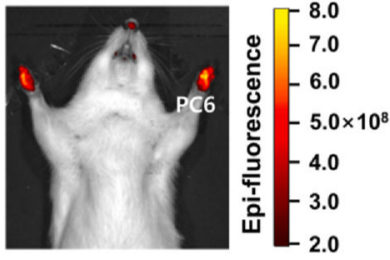

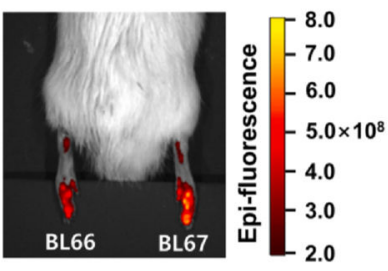

Radiant Efficiency $\frac{\mathrm{p} / \mathrm{sec} / \mathrm{cm}^{2} / \mathrm{sr}}{\mu \mathrm{M} / \mathrm{cm}^{2}}$

Fig. 1.

Neurogenic inflammation at acupoints in IMH rats. A. Schematic showing the experimental procedure used in the rat model of immobilization-induced hypertension (IMH). B.

Development of hypertension following immobilization restraint stress. In restrained rats, systolic blood pressure was significantly elevated, reaching a level consistent with hypertension of over $150 \mathrm{mmHg}$ within $1 \mathrm{~h}$, compared to that in un-restrained control rats (Control). Asterisks $* * *$ indicate significance level $\mathrm{p}<0.001$ vs. Control. C. The change in blue color at a neurogenic spot (Neuro-Sp) in restrained rats. Asterisks $* * *$ indicate significance level $\mathrm{p}<0.001$ vs. Control. D. Representative images of blue color changes at Neuro-Sps in an IMH rat. E. Representative images of the in vivo fluorescence of Neuro-Sps using the IVIS Lumina III system in normal (Control) or IMH rats (Hypertension). 

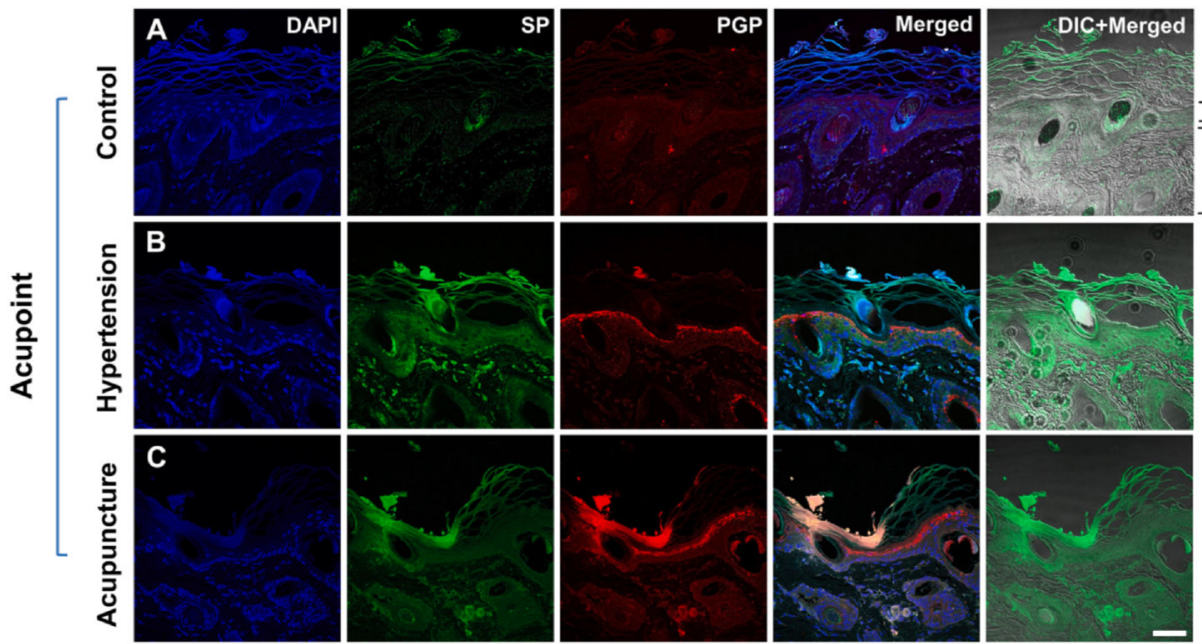

Jepidermis

dermis

Fig. 2.

Increased SP expression at neurogenic spots. A-C. Immunostaining for DAPI, SP, and PGP 9.5 at normal skin in naïve rats (A), acupoints (Neuro-Sps) in IMH rats (B), and acupuncture-stimulated skin in naïve rats $(C)$. Increased SP (green) expression was found at Neuro-Sps in IMH rats (B) and acupuncture-stimulated skin in naïve rats (C). DAPI, blue; PGP 9.5-positive structures, red; DIC, differential interference contrast. Bar $=50 \mu \mathrm{m}$. D. Quantification of SP-stained areas (green) in the Neuro-Sps of the wrists of control rats (Control, $\mathrm{n}=6$ ) and IMH rats (Hypertension, $\mathrm{n}=6$ ) and the acupuncture-stimulated skins (Acupuncture, $\mathrm{n}=6$ ). Asterisks $* * *$ signify significance level $\mathrm{p}<0.001$ vs. Control. 

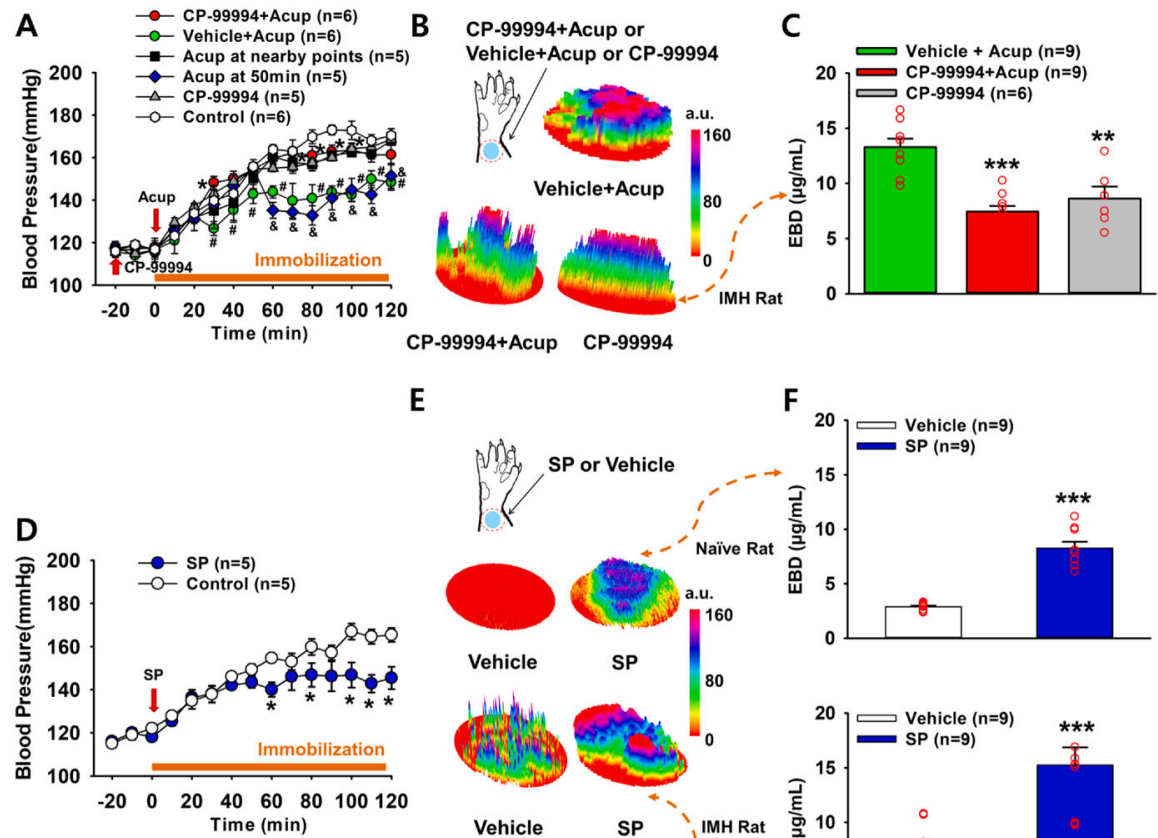

$\mathbf{E}$

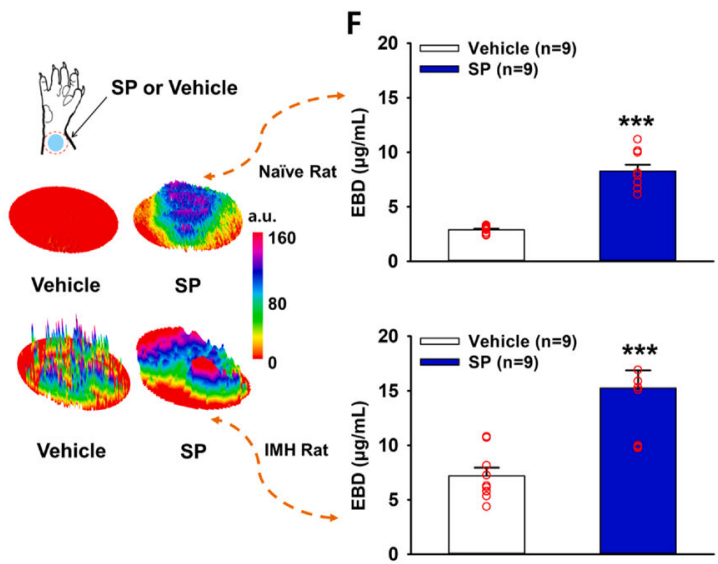

$\mathrm{H}$

I
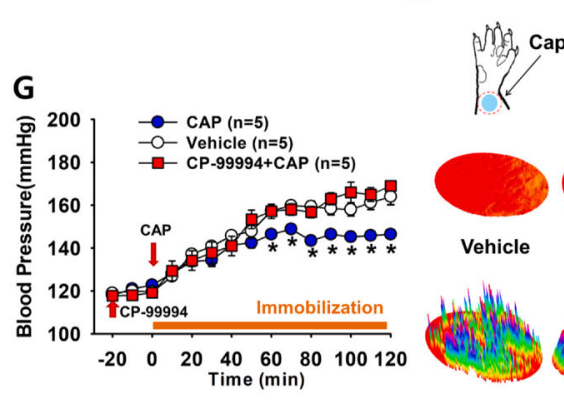

Vehicle

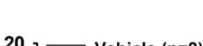

Fig. 3.

Effect of neurogenic spot stimulation on the development of hypertension. A-C. Effect of pretreatment with CP-99994, an SP receptor antagonist, on the acupuncture-induced suppression of hypertension in IMH rats. Acupuncture prevented the development of hypertension following immobilization (Vehicle + Acup) compared to that observed in the control (IMH only). This effect was abolished by the injection of CP-99994 into Neuro-Sps 20 min before acupuncture (CP-99994 + Acup) (A). Hashtag \# and ampersand \& indicates significance level $\mathrm{p}<0.05$ vs. Control (immobilization only); Asterisk * indicates significance level $\mathrm{p}<0.05$ vs. Vehicle + Acup. Acup, acupuncture; CP-99994, injection of CP-99994 into Neuro-Sps 20 min without acupuncture; Acup at nearby points, acupuncture at SI5 $5 \mathrm{~mm}$ apart from PC6. Acup at $50 \mathrm{~min}$, acupuncture at $50 \mathrm{~min}$ during the development of hypertension following immobilization. Three-dimensional (3D) images of Neuro-Sps over the skin of the wrist in vehicle + Acup-, CP-99994 + Acup- or CP-99994-treated rats 
(B) and the concentration of Evans blue dye (EBD) in the skin in the wrist in vehicle + Acup-, CP-99994 + Acup- or CP-99994-treated IMH rats (C). Asterisks *** indicated significance level $p<0.001$ and $* *$ indicated significance level $p<0.01$ vs. Vehicle + Acup. D-F. Effect of subcutaneous SP at Neuro-Sps on blood pressure in IMH rats. Injection of SP into the skin of the Neuro-Sps reduced the development of hypertension compared to the injection of vehicle in IMH rats (D). Asterisk * indicates significance level $\mathrm{p}<0.05$ vs.

Vehicle. 3D images of Neuro-Sps at the wrist acupoints (skin) in vehicle- or SP-treated naïve and IMH rats (E) and the concentration of EBD in the skin in the wrist of vehicle- or SPtreated naïve and IMH rats (F). Asterisks $* * *$ indicate significance level $\mathrm{p}<0.001 \mathrm{vs.}$ Vehicle. G-I. Effect of the subcutaneous injection of capsaicin into Neuro-Sps on blood pressure in IMH rats. Injection of capsaicin (CAP) into Neuro-Sps suppressed the development of hypertension compared to the injection of vehicle in IMH rats, and this effect was abolished by the injection of CP-99994 into Neuro-Sps 20 min before injection of CAP (CP-99994 + CAP) (G). Asterisk * indicates significance level $\mathrm{p}<0.05$ vs. Vehicle. 3D images of Neuro-Sps in wrist acupoints (skin) in vehicle- or CAP-treated naïve and IMH rats $(\mathrm{H})$ and the concentration of EBD in the skin in the wrist of vehicle- or CAP-treated naïve and IMH rats (I). Asterisks ** indicate significance levels $\mathrm{p}<0.01$, *** $=\mathrm{p}<0.001$ vs. Vehicle. a.u. $=$ arbitrary unit. 

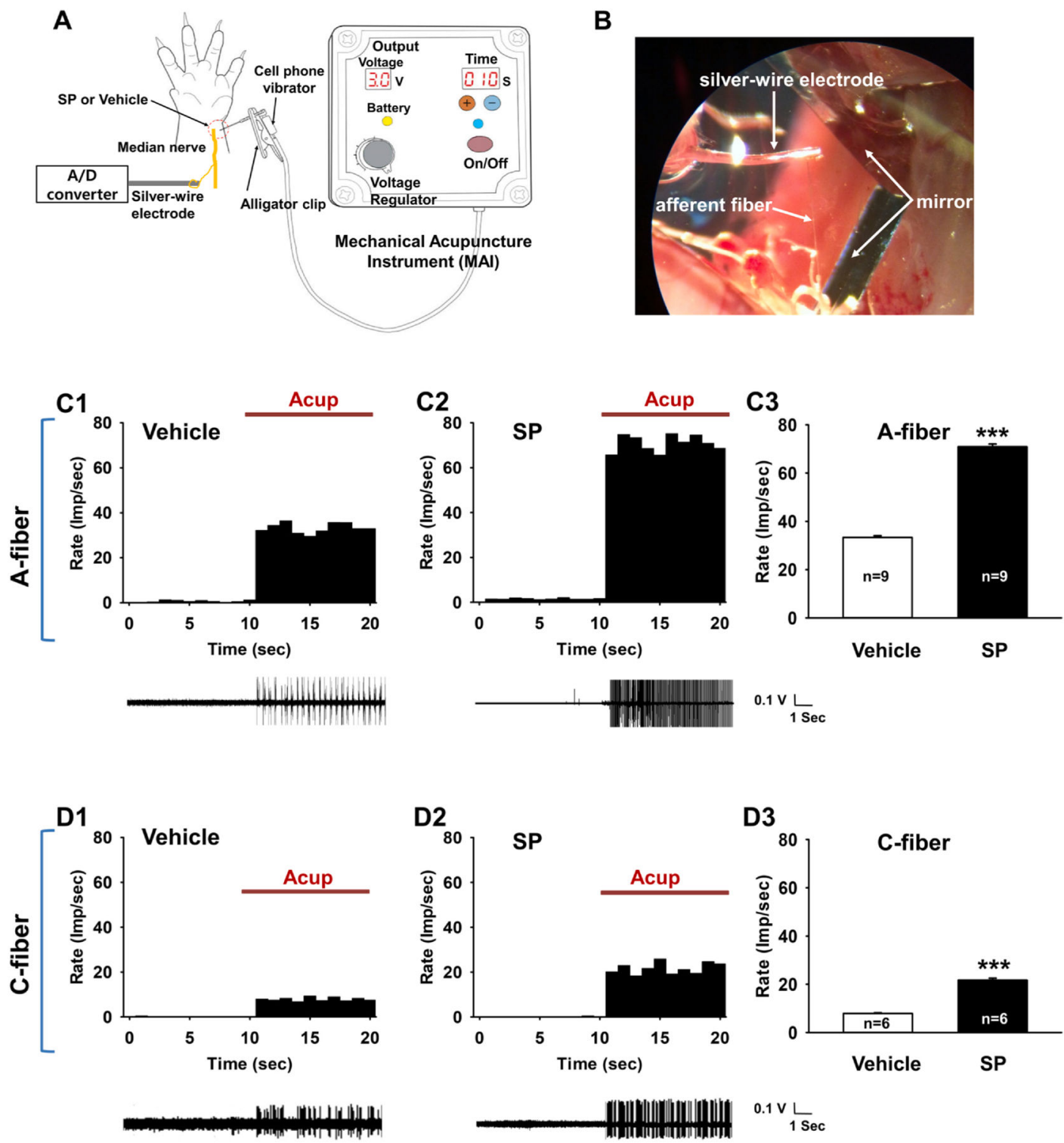

Fig. 4.

Response of A- or C-fibers to acupuncture stimulation following intradermal SP injection in normal rats. A. Schematic showing the procedure used during acupuncture with the mechanical acupuncture instrument (MAI) and single-fiber recordings. B. A representative image of single-fiber recording. C. Response of A-fibers to acupuncture stimulation following subcutaneous SP injection. A representative histogram (upper) and afferent discharges (lower) from the single-unit recording of A-fibers during acupuncture stimulation in vehicle- (saline, $\mathrm{n}=9, \mathrm{C} 1)$ and SP-injected $(\mathrm{n}=9, \mathrm{C} 2)$ groups. Mean firing rates of Afibers during acupuncture stimulation of Neuro-Sps (PC6 area) in saline- or SP-injected groups (C3). imp/sec, impulse per second; Asterisks *** indicate significance level $\mathrm{p}<$ 0.001 vs. Vehicle. D. Response of C-fibers to acupuncture stimulation following intradermal SP injection. A representative histogram (upper) and afferent discharges (lower) from the single-unit recording of C-fibers $(n=6)$ during acupuncture stimulation in saline-injected $(n$ 
$=6, \mathrm{D} 1)$ and SP-injected $(\mathrm{n}=6, \mathrm{D} 2)$ groups. Mean firing rates of C-fibers during acupuncture stimulation in saline- or SP-injected groups (D3). Asterisks *** indicate significance level $\mathrm{p}<0.001$ vs. Vehicle. 


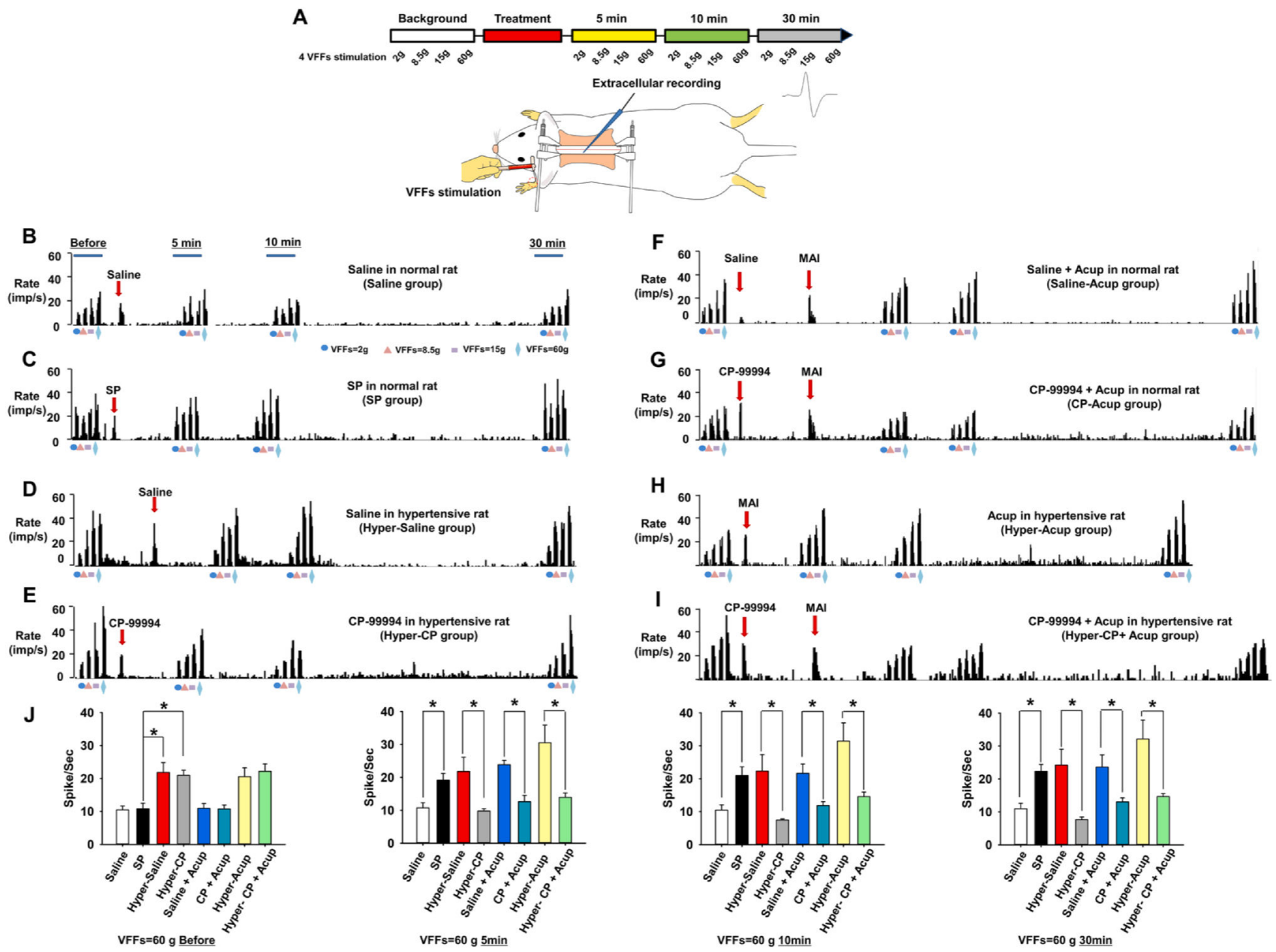

Fig. 5.

Response of spinal WDR neuronal activity to intradermal SP injection. A. Experimental schedule (upper) and schematic of extracellular recording in the spinal dorsal horn (lower). Four different VFFs were applied to the skin over the wrist at before and $5 \mathrm{~min}, 10 \mathrm{~min}$ and 30 min after treatment (vehicle, SP or CP-99994). VFFs, von Frey filaments. B-C. The neuronal activity of WDR neurons in response to non-noxious (VFFs: 2, 8.5, and $15 \mathrm{~g}$ ) and noxious (VFF: $60 \mathrm{~g}$ ) mechanical stimulation of somatic fields after subcutaneous saline injection in normal rats (Saline group, $\mathrm{n}=11$; B) or subcutaneous SP injection in normal rats (SP group, $n=9 ; C$ ). D-E. The neuronal activity of WDR neurons in response to nonnoxious (VFFs: 2, 8.5, and $15 \mathrm{~g}$ ) and noxious (VFF: $60 \mathrm{~g}$ ) mechanical stimulation of somatic fields after either saline (Hyper-Saline group, $n=6$; D) or CP-99994 injection in IMH rats (Hyper-CP group, $\mathrm{n}=8$; E). F-G. The neuronal activity of WDR neurons in response to nonnoxious (VFFs: 2, 8.5, and $15 \mathrm{~g}$ ) and noxious (VFF: $60 \mathrm{~g}$ ) mechanical stimulation of somatic fields after subcutaneous saline/CP-99994 injection with acupuncture. Acupuncture treatment was performed by using a mechanical acupuncture instrument (MAI) in salineinjected rats (Saline-Acup group, $\mathrm{n}=6$; F) or subcutaneous CP-99994 injected rats (CPAcup group, $\mathrm{n}=7$; G). H-I. The neuronal activity of WDR neurons in response to nonnoxious (VFFs: 2, 8.5, and $15 \mathrm{~g}$ ) and noxious (VFF: $60 \mathrm{~g}$ ) mechanical stimulation of somatic 
fields after acupuncture (Hyper-Acup, $\mathrm{n}=6$; H) or subcutaneous CP-99994 injection with acupuncture (Hyper-CP + Acup group, $n=6$; I) in IMH rats. J. Mean firing rates of WDR neurons in response to $60 \mathrm{~g}$ VFF stimuli for $10 \mathrm{sec}$ each at before and $5 \mathrm{~min}, 10 \mathrm{~min}, 30 \mathrm{~min}$ after saline (Saline) or SP (SP) administration in normal rats, saline (Hyper-Saline) or CP-99994 (Hyper-CP) administration in IMH rats, saline + acupuncture (Saline-Acup) or CP-99994 + acupuncture (CP-Acup) treatment in normal rats or acupuncture (Hyper-Acup) or CP-99994 + acupunctrure (Hyper-CP + Acup) treatment in IMH rats. Asterisk * indicates significance level $\mathrm{p}<0.05$. 

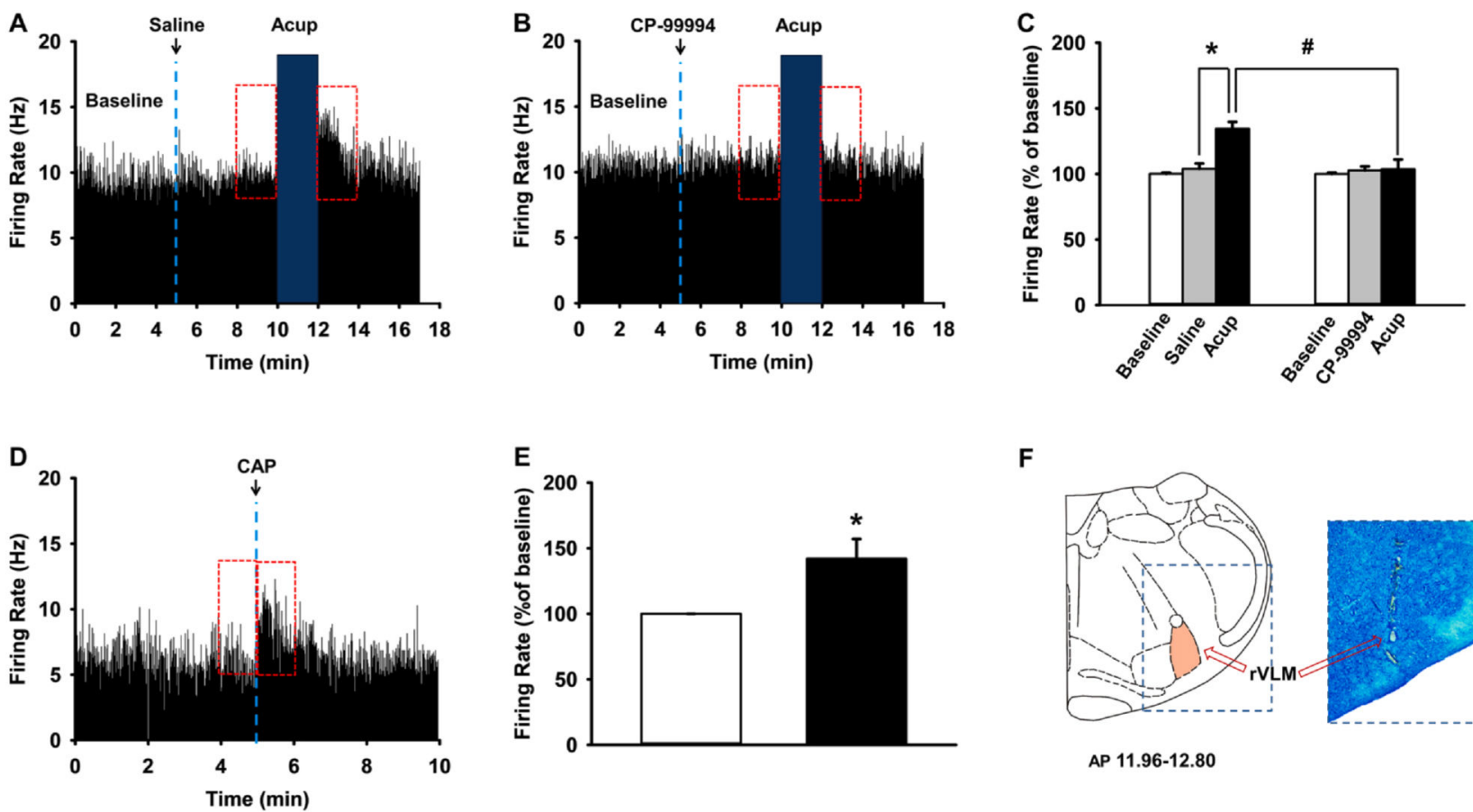

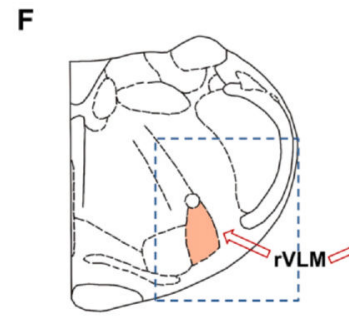

AP $11.96-12.80$

Fig. 6.

Effect of local injection of an SP receptor antagonist, CP-99994, into Neuro-Sps on responses of rVLM neurons to MAI acupuncture. A-C. Effect of local injection of an SP receptor antagonist, CP-99994, into Neuro-Sps on responses of rVLM neurons to MAI acupuncture. Representative histograms showing rVLM neuronal activity following MAI acupuncture (A) and pretreatment of CP-99994 + MAI acupuncture (B). C. Mean values of firing rates/sec during 2 min after intradermal saline/CP-99994 and MAI at Neuro-Sps, expressed as percentage of the pretreatment values (Baseline). Asterisk * indicates significance level $\mathrm{p}<0.05$, and hashtag \# $\mathrm{p}<0.05$. MAI stimulation at PC6 near wrist increased the firing rates of rVLM neurons $(\mathrm{n}=8 ; \mathrm{A}, \mathrm{C})$ in IMH rats, while pretreatment of SP receptor antagonist CP-99994 prior to acupuncture prevented acupuncture-induced activation of rVLM neurons in IMH rats $(\mathrm{n}=8, \mathrm{~B}, \mathrm{C})$. D, E. Effect of intradermal injection of capsaicin into Neuro-Sps on rVLM neuronal activities in normal rats. In vivo extracellular recordings from rVLM neurons (D) and mean values of firing rates/sec during 1 min before and after intradermal injection of capsaicin into the wrist Neuro-Sps, expressed as percentage of the pretreatment values (Baseline) (E). Asterisk *p indicates significance level $\mathrm{p}<0.05$. F. A representative picture of rVLM lesion stained by toluidine blue. 